\title{
Pregnancy complicated by HTLV-1-associated myelopathy/tropical spastic paraparesis (HAM/TSP): a case report of one woman over three consecutive courses of pregnancy and childbirth
}

\author{
Hiroshi Mori ${ }^{1}$, Eiji Shibata ${ }^{1}$, Tomoichiro Kuwazuru ${ }^{1}$, Takayuki Uchimura ${ }^{1}$, Emi Kondo ${ }^{1}$, \\ Yukio Iwanaka ${ }^{1}$, and Kiyoshi Yoshino ${ }^{1}$ \\ ${ }^{1}$ University of Occupational and Environmental Health Japan
}

April 24, 2021

\begin{abstract}
There are few reports on pregnancy and childbirth of HTLV-1-associated myelopathy/tropical spastic paraparesis (HAM/TSP) patients. We present a woman who diagnosed with HAM/TSP and used a wheelchair in daily life. She had no neurological impairments and no obstetrical complications during her three courses of pregnancy and childbirth.
\end{abstract}

Title page:

Title:

Pregnancy complicated by HTLV-1-associated myelopathy/tropical spastic paraparesis (HAM/TSP): a case report of one woman over three consecutive courses of pregnancy and childbirth

Authors:

Hiroshi Mori MD ${ }^{1) 2}$ hmori-1@med.uoeh-u.ac.jp

Eiji Shibata MD PhD ${ }^{3)}$ age-s@med.uoeh-u.ac.jp

Tomoichiro Kuwazuru MD ${ }^{3)}$ t-kuwazuru@med.uoeh-u.ac.jp

Takayuki Uchimura MD ${ }^{1)}$ t-uchimura@med.uoeh-u.ac.jp

Emi Kondo MD ${ }^{1)}$ ek89224@med.uoeh-u.ac.jp

Yukio Iwanaka MD ${ }^{4}$ iwanaka@clnc.uoeh-u.ac.jp

Kiyoshi Yoshino MD PhD ${ }^{3)}$ k-yoshino@med.uoeh-u.ac.jp

\section{Affiliations:}

1. Department of Perinatal Medical Center, School of Medicine, University of Occupational and Environmental health

2. Department of Microbiology, Graduate school of medicine, University of Occupational and Environmental health

3. Department of Obstetrics and Gynecology, School of Medicine, University of Occupational and Environmental health

4. Department of Neurology, School of Medicine, University of Occupational and Environmental health 


\section{Corresponding author:}

Eiji Shibata

Department of Obstetrics and Gynecology, University of Occupational and Environmental health, 1-1 Iseigaoka, Yahatanishi-ku, Kitakyushu-city, Fukuoka 807-8555, Japan

Telephone No.: +81-93-603-1611

Fax No.: +81-93-691-9337

Email address: age-s@med.uoeh-u.ac.jp

\section{Key Clinical Message (47 words)}

There are few reports on pregnancy and childbirth of HTLV-1-associated myelopathy/tropical spastic paraparesis (HAM/TSP) patients. We present a woman who diagnosed with HAM/TSP and used a wheelchair in daily life. She had no neurological impairments and no obstetrical complications during her three courses of pregnancy and childbirth.

\section{Keywords}

HTLV-1, HTLV-1-associated myelopathy/tropical spastic paraparesis, pregnancy

\section{Introduction}

All over the world, five to ten million people are infected with the human T-cell lymphotropic virus type1 (HTLV-1 $)^{1)}$, approximately $0.25 \%$ to $3.8 \%$ of HTLV-1 carriers will develop HTLV-1 associated myelopathy/ tropical spastic paraparesis $(\mathrm{HAM} / \mathrm{TSP})^{2)}$. HAM/TSP is a slowly progressive type of myelopathy and is characterized by spastic paraparesis, bladder and sphincter dysfunction, and mild sensory impairment. Approximately $80 \%$ of patients with HAM progress slowly after the onset. Nearly $20 \%$ of patients with HAM/TSP progress rapidly after onset and become unable to walk within 2 years, requiring a wheelchair in daily life. The average age at onset is around 50-year-old, there are few reports of pregnant women with $\mathrm{HAM} / \mathrm{TSP}$.

A nationwide HTLV-1 screening program for pregnant women was started in 2011 in Japan, and we believe that the incidence of HTLV-1 careers will decrease in the future. Screening for all pregnant women and provision of information to HTLV-1 careers have been systematized in Japan, there is little information on pregnancy and childbirth of patients with HAM/TSP who develop from HTLV-1 carriers.

There are more patients with HAM in women than in men, and several publications are pointing to early juvenile HAM/TSP. For women with HAM/TSP in reproductive age and their family, for medical staff, we think it is important to report pregnancy and childbirth of patients with HAM/TSP.

We report here of one woman with HAM/TSP and her three consecutive courses of pregnancy and childbirth without severe complications on her and her baby. These findings suggest that women with HAM/TSP, even those who have spastic paraparesis and use a wheelchair in daily life, should not be discouraged from pregnancy.

\section{Case history}

\section{History of present illness}

Written informed consent was obtained to report the case.

When she was 15-year-old, she found something wrong with her inguinal region while exercising. She gradually became difficult to move her both lower limbs. She had difficulty in walking, so she went to the neurology department at the age of 19 .

\section{Family history}


maternal grandmother; ATL, maternal grandfather; hypertension, mother; hypertension

\section{Laboratory findings}

Somatosensory evoked potential (tibial nerve stimulation); right $51.0 \mathrm{~ms}$, left $48.0 \mathrm{~ms}(32.6-40.8 \mathrm{~ms})$

Magnetic stimulation motion evoked potential; right $44.1 \mathrm{~ms}$, left $43.2 \mathrm{~ms}(25.0-30.8 \mathrm{~ms})$

Central motor conduction time (CMCT); right $26.2 \mathrm{~ms}$, left $27.8 \mathrm{~ms}(7.6-13.2 \mathrm{~ms})$

MRI (cervical and thoratic spine); no abnormality

Serum anti-HTLV-1 antibody; positive

Cerebrospinal fluid anti-HTLV-1 antibody; positive (512 times)

Cerebrospinal fluid IgG index; increased

Based on the above results and physical exam, she was diagnosed with HAM/TSP.

At the age of 20, one year after the initial diagnosis, aggravation of gait disorder appeared, steroid pulse therapy was performed with a diagnosis of acute exacerbation. Her gait was stable and she was discharged and outpatient managed.

Her symptoms of spastic paraplegia gradually worsened, and two years after her initial diagnosis, she became unable to walk on her own and began to use a wheelchair in daily life. At the same time, she had dysuria and began to do self-intermittent catheterization for bladder dysfunction. And she had had a urinary tract infection (UTI) twice before her first pregnancy.

\section{Courses of pregnancy and childbirth}

A 24-year-old, G1P0, pregnant woman diagnosed with HAM/TSP presented at our hospital at 12 weeks of gestation.

Her neurological physical findings at the first our visit; bilateral lower limbs; spastic paraparesis, Osame's motor disability score (OMDS): 11, limb tendon reflex hyperreflexia, Babinski's reflex positive.

She used a wheelchair in daily life for both lower limbs paraparesis and did self-intermittent catheterization for bladder dysfunction.

There were no obstetric abnormalities and no exacerbation of neurological findings in the first course of pregnancy.

She wore graduated compression stocking in daily life and she conducted physical therapy by her husband (his profession was physical therapists) for prevention of venous thromboembolism (VTE) during pregnancy.

She, with a gestational age of 38 weeks and 5 days, was admitted to our hospital because of spontaneous labor with cervical dilation of $5 \mathrm{~cm}$. We performed augmentation of labor with the use of intravenous oxytocin for the treatment of prolonged labor in the first stage of labor. The second stage of labor was also prolonged, and she could not push down, we performed vacuum extraction delivery. The birth weight of the neonate was 3,128g with umbilical cord $\mathrm{pH} 7.25$, and the 1-minute and 5-minute Apgar scores were 8/9.

Her labor had lasted 21 hours, and postpartum blood loss was estimated at $686 \mathrm{ml}$.

In postpartum period, she received current standard non-pharmacological VTE prophylaxis recommendations; she also wore graduated compression stocking and conducted physical therapy by he husband. She did not have a VTE during pregnancy, peripartum and postpartum period.

We explained that breastfeeding is at high risk of mother-to-child transmission of HTLV-1, she opted for short-term (3months) breastfeeding and formula-milk nutrition. 
Following two consecutive courses of pregnancies after the first pregnancy and childbirth were uneventful. We show the summary of these three courses (Table1). Outcome and follow-up

There was no impairment of neurological findings after the three courses of pregnancy and childbirth.

To prevent an infection of HTLV-1 via breastfeeding, with the patient consent, the first and second babies were fed by short-term ( 3 months) breastfeeding and formula-milk, the third baby was fed by formula-milk only. We have been monitoring the babies for the infection until they are two-year-old. Three children have not been infected with HTLV-1 until now.

\section{Discussion}

We report a case of one woman over three consecutive courses of pregnancy and childbirth complicated with HAM/TSP. There is no evidence-based medical literature on managing pregnancy and childbirth for pregnancies complicated by HAM/TSP. There are very few reports of pregnancy complicated by HAM/TSP, in the first place. For this reason, women with HAM/TSP in reproductive age and their family may hesitate to get pregnant.

Regarding pregnancy and childbirth management in HAM/TSP patients, we need to consider the followings: (1) fertility, (2) the impact of pregnancy and childbirth on the disease, (3) the impacts of the disease on the course of pregnancy and childbirth, (4) prevention of mother-to-child HTLV-1 transmission, (5) maternal VTE prophylaxis.

\section{Fertility}

There are limited data on the impact of HTLV-1 on fertility. In some reports, no differences were found in fertilization among the prevalence of HTLV-1 infection ${ }^{6)}$. There is no data on the impact of patients with $\mathrm{HAM} / \mathrm{TSP}$ on fertility.

\section{The impact of pregnancy and childbirth on the disease}

Although there are no study regarding patients with HAM/TSP in pregnant women have been reported, there is a potential for pregnancy to impact HAM/TSP. High HTLV-1 proviral load (PVL) levels have been evaluated as important predictors of development of ATL\{Rebecca J. Baer, 2021 \#26; Iwanaga M, 2010 $\# 233^{7}$ ) HTLV-1 PVL levels plateau during pregnancy and increase after delivery ${ }^{8)}$. In this case, we could not evaluate her HTLV-1 PVL levels. It is unclear that whether postpartum HTLV-1 PVL levels elevation is transient or persistent at this moment. She had three consecutive courses of pregnancy and childbirth within four years, but it is unclear if it would be a problem to get pregnant soon after giving birth.

The impacts of the disease on the course of pregnancy and childbirth

Constipation and dysuria, and gait disturbance are possible impacts of the disease on the course of pregnancy and childbirth. These symptoms may be impaired by physical and physiological changes in pregnancy (uterine enlargement, maternal weight gain, decrease in intestinal peristalsis due to endocrinological changes). In this case, she had constipation trouble during pregnant period, while there was no impairment before pregnancy. Neurogenic bladder is commonly observed in HAM/TSP, results in increased UTI rates. It can induce preterm birth, we should diagnose it earlier with a physical exam and microscopic exam of urine. In this case, she had been treated for UTI twice before the first pregnancy, we thought that she was more likely to get a UTI during pregnancy and postpartum period than before pregnancy. Conversely, She did not have a UTI and she gave birth in term during her three courses of pregnancy and childbirth.

\section{Prevention of mother-to-child HTLV-1 transmission}

To prevent an infection of HTLV-1 via breastfeeding, with the patient consent, the first and second babies were fed by short-term (3 months) breast-feeding and formula-milk, the third baby was fed by exclusive formula-milk. In HTLV-1-endemic areas, to prevent mother-to-child transmission, we should inform mothers and their family that extended breastfeeding links increasing the risk of infection. The rate of infection is 
$15.7 \%$ among children who have been breastfeeding for more than 12 months. The mother-to-child transmission rate of complete formula-milk is $0-5.6 \%$, while of breastfeeding period of six-seven months or more is $\left.11.3-33.3 \% .^{9}\right)$ It is clear that the latter has a high infection rate. In some retrospective study in Japan, the mother-to-child transmission rate with short-term breast-feeding ( 3 months) was 1.6-8.5\%, and its risk ratio compared with that of exclusive formula feeding was not statistically different. ${ }^{9,10)}$ Even if the mother wishes to breastfeeding, it is desirable that she should stop it within three months.

\section{Maternal VTE prophylaxis}

We needed to consider about maternal VTE prophylaxis. In general, wheelchair use in daily life is a risk factor for developing VTE, and is at higher risk during pregnancy and postpartum period. In this case, she had never been treated for VTE before first pregnancy and her husband was physical therapist, we thought that she did not require anticoagulation agents such as unfractionated heparin during pregnancy. We told her prophylaxis for VTE, to wear graduated compression stocking and to be conducted aggressive physical therapy by her husband in daily life. She did not have a VTE during her three courses of pregnancy and childbirth and postpartum period.

We report here of one woman with HAM/TSP and her three consecutive courses of pregnancy and childbirth without complications on her and her baby. These findings suggest that women with HAM/TSP, even those who have spastic paraparesis and use a wheelchair in daily life, should not be discouraged from pregnancy.

\section{Reference}

1. Gessain A, Cassar. 2012. Epidemiological aspect and world distribution of HTLV-1 infection. Front. Microbiol 3:388.

2. Yamano Y, Sato T. 2012. Clinical pathophysiology of human T-lymphotropic virus-type 1-associated myelopathy/tropical spastic paraparesis. Front Microbiol 3:389

3. Nakagawa M, Izumo S, Ijichi S, et al. 1995. HTLV-1-associated myelopathy : Analysis of 213 patients based on clinical features and laboratory findings. J Neurovirol 1:50-61

4. Yonemoto N, Suzuki S, Sekizawa A, Hishi S, Sagara Y, Itabashi K. 2020. Implementation of nationwide screening of pregnant women for HTLV-1 infection in Japan: analysis of a repeated cross-sectional study. BMC public health 20:1150

5. A Schwalb, V Perez-Muto, R Cachay, et al. 2020 Early-Onset HTLV-1-Asssociated Myelopathy/Tropical Spastic Paraparesis. Pathogens 7;9(6):450

6. Torshizi MM, Khalighi AR, Isram MF, et al. 2014. Effect of human T-cell lymphotrophic virus type 1 (HTLV-1) in seropositive infertile women on intracytoplasmic sperm injection (ICSI) outcome. Iran J Reprod Med 12:15-18

7. M Iwanaga, T Watanabe, A Utsunomiya, et al. 2010. Human T-cell leukemia virus type 1 (HTLV1) proviral load and disease progression in asymptomatic HTLV-1 carriers: a nationwide prospective study in Japan. Blood 26;116(8):1211-9

8. N Fuchi, K Miura, T Tsukiyama, et al. 2018. Natural Course of Human T-cell Leukemia Virus Type 1 Proviral DNA Levels in Carriers During Pregnancy. J Infect Dis 217:1383-1389

9. Itabashi K, Miyazawa T, Sekizawa A, et al. 2020. A nationwide antenatal human T-cellleukemia virus type-1 antibody screening in Japan. Front Microbiol 11:595

10. Itabashi K, Miyazawa T, Nerome Y, et al. 2021. Issues on infant feeding for postnatal prevention of human T-cell leukemia/lymphoma virus type-1 mother-to-child transmission. Pediatr Int 63(3):284289.

\section{Acknowledgements}

We would like to thank for the medical staffs of department of perinatal medical center and obstetrics and gynecology, school of medicine, University of Occupational and Environmental health.

\section{Conflict of interest}

None declared 


\section{Author contributions}

HM: involved in management of pregnancy and labor and wrote the manuscript. TK, TU and EK: involved in management of labor. YI: involved in management of neurological symptoms during pregnancy. ES and KY: supervised the project. All authors: discussed the results and contributed to the final manuscript.

\section{Figure legends}

Table1. Delivery outcomes and postpartum management

ART: assisted reproductive technologies, FM: formula-milk, GCS: graduated compression stocking, NVD:

Normal vaginal delivery, PT: physical therapy, STBF: short-term (3 months) breastfeeding, VE: vacuum extraction, VTE: venous thromboembolism

\section{Hosted file}

20210419 HAM Tables.pdf available at https://authorea.com/users/385829/articles/519362pregnancy-complicated-by-htlv-1-associated-myelopathy-tropical-spastic-paraparesisham-tsp-a-case-report-of-one-woman-over-three-consecutive-courses-of-pregnancy-andchildbirth 